\title{
Left and Right in small-x neutrino DIS
}

\author{
Vladimir Zoller* \\ ITEP, B. Cheremushkinskaya 25, 117218 Moscow, Russia \\ E-mail: zoller@itep.ru
}

\begin{abstract}
The color dipole analysis of nuclear effects in charge current DIS is presented. The emphasis is put on the pronounced effect of left-right asymmetry of shadowing in neutrino-nucleus DIS at small values of Bjorken $x$. Strikingly different scaling behavior of nuclear shadowing for the left-handed and right-handed $W^{+}$is predicted. Large, about $25 \%$, shadowing in the $\mathrm{Fe}$ structure functions is predicted, which is important for a precise determination of the P-odd nucleon structure functions $x F_{3}^{v(\bar{v})}$ and $\Delta x F_{3}=x F_{3}^{v}-x F_{3}^{\bar{v}}$.
\end{abstract}

Diffraction 06, International Workshop on Diffraction in High-Energy Physics September 5-10, 2006

Adamantas, Milos island, Greece

${ }^{*}$ Speaker. 


\section{Introduction}

Responsible for the account of the left-right asymmetry effects in diffractive neutrino interactions with nuclear medium is the parity-odd structure function $F_{3}$ which appears in the standard decomposition of the forward amplitude for virtual W-nucleon scattering [1]

$$
\frac{1}{\pi} W_{\mu \nu}=\ldots+i \varepsilon_{\mu v \lambda \rho} \frac{p_{\lambda} q_{\rho}}{2 p q} F_{3}\left(x, Q^{2}\right) \ldots,
$$

where $p$ and $q$ are the target nucleon and the virtual $W^{+}$-boson four-momenta, respectively. The physical picture behind the decomposition (1.1) depends on the choice of particular reference frame. Indeed, in the brick wall frame, where the parton picture of a nucleon is manifest, in the chiral limit the left-handed W-boson $\left(W_{L}\right)$ interacts only with quarks while the right-handed $W_{R}$ interacts only with anti-quarks. Hence, the representation for $F_{3}$ in terms of small-x parton densities [1]

$$
\begin{gathered}
F_{3}^{v N}=2(s-\bar{c}) \\
F_{3}^{\bar{V} N}=2(c-\bar{s}) .
\end{gathered}
$$

Thus, the isospin symmetry of the nucleon sea implies that at small Bjorken $x$ the structure function $F_{3}$ is dominated by the charm-strange weak current.

In the dipole/laboratory frame the small- $x$ DIS is treated in terms of the interaction of the quark-antiquark color dipole (CD) of size $\mathbf{r}$, that the virtual $W$ transforms into, with the target nucleon $[2,3]$. This interaction is described by the universal flavor-independent color dipole cross section $\sigma(x, r)$. At small $x$ the dipole size $\mathbf{r}$ is a conserved quantum number and the parity nonconservation effect can be quantified in terms of CD sizes of left-handed and right-handed Wbosons. Hence, the representation of $F_{3}$ in terms of absorption cross sections for $W_{L}$ - and $W_{R}$-bosons $\left(\sigma_{L, R}\right)$

$$
2 x F_{3}\left(x, Q^{2}\right)=\frac{Q^{2}}{4 \pi^{2} \alpha_{W}}\left(\sigma_{L}-\sigma_{R}\right)
$$

where $\alpha_{W}=G_{F} m_{W}^{2} / 4 \pi \sqrt{2}$. These cross sections are calculated as a quantum mechanical expectation values of $\sigma(x, r)$. For small dipoles

$$
\sigma(x, r) \propto r^{2} \alpha_{S}\left(C / r^{2}\right) G\left(x, C / r^{2}\right)
$$

where $C \simeq 10$ and $G\left(x, \kappa^{2}\right)$ is the gluon density in the proton. Then, the deviation of $F_{3}$ from zero implies that dipole sizes of left-handed and right-handed W-bosons are different. The origin of this difference is of course the left-handedness of weak currents. The latter makes the light-cone densities of charm-strange dipoles highly asymmetric functions of the Sudakov variable $z[4,5]$,

$$
\left|\Psi_{L}(z, r)\right|^{2} \sim z^{2} \varepsilon^{2} K_{1}^{2}(\varepsilon r)
$$

and

$$
\left|\Psi_{R}(z, r)\right|^{2} \sim(1-z)^{2} \varepsilon^{2} K_{1}^{2}(\varepsilon r)
$$

Hereafter $z$ stands for the momentum fraction of the light-cone $W$-boson carried away by the $c$ quark. Characteristic values of $z$ for left-handed and right-handed $c \bar{s}$ states are very different. 
Besides, the dipole sizes are limited from above by the factor $K_{1}^{2}(\varepsilon r) \sim \exp (-2 \varepsilon r)$ and the cutoff parameter $\varepsilon$ depends on $z$,

$$
\varepsilon^{2}=z(1-z) Q^{2}+(1-z) m_{c}^{2}+z m_{s}^{2} .
$$

Therefore, different $z$ correspond to different dipole sizes dominating the integrals for the dipolenucleon cross sections $\sigma_{L}$ and $\sigma_{R}$ at $Q^{2} \gg m_{c}^{2}$,

$$
\begin{array}{r}
\sigma_{L}\left(x, Q^{2}\right)=\int_{0}^{1} d z \int d^{2} \mathbf{r}\left|\Psi_{L}(z, \mathbf{r})\right|^{2} \sigma(x, r) \\
\propto \frac{1}{Q^{2}} \int_{1 / Q^{2}}^{1 / m_{s}^{2}} \frac{d r^{2}}{r^{4}} \sigma(x, r) \\
\propto \frac{1}{Q^{2}} \int_{1 / Q^{2}}^{1 / m_{s}^{2}} \frac{d r^{2}}{r^{2}} \alpha_{S}\left(r^{2}\right) G\left(x, 10 / r^{2}\right) \propto \frac{1}{Q^{2}} \log \frac{Q^{2}}{m_{s}^{2}} \\
\propto \frac{1}{Q^{2}} \int_{1 / Q^{2}}^{1 / m_{c}^{2}} \frac{d r^{2}}{r^{2}} \alpha_{S}\left(r^{2}\right) G\left(x, 10 / r^{2}\right) \propto \frac{1}{Q^{2}} \log \frac{Q^{2}}{m_{c}^{2}} .
\end{array}
$$

where $m_{c}$ and $m_{s}$ are constituent quark masses. In Eqs.(1.9, 1.10) it has been taken into account that the product $\alpha_{S}\left(r^{2}\right) G\left(x, C / r^{2}\right)$ is approximately flat in $r^{2}$.

The mirror asymmetry of diffractive interactions of electroweak bosons with nuclear matter is enhanced by the large thickness of a nucleus [5, 6]. In particular, the nuclear shadowing effect appears to be very different for left-handed and right-handed $W$-bosons. The point is that the shadowing term $\delta \sigma_{A, \lambda}$, where $\lambda=L, R=-1,+1$, in expansion of the nuclear cross section

$$
\sigma_{A, \lambda}=A \sigma_{\lambda}-\delta \sigma_{A, \lambda}
$$

is the quadratic functional of the $\mathrm{CD}$ cross section. To the lowest order in $\sigma T$ this term reads

$$
\delta \sigma_{A, \lambda} \simeq \frac{\pi}{4}\left\langle\sigma^{2}\right\rangle_{\lambda} \int d b^{2} T^{2}(b)
$$

Here $T(\mathbf{b})=\int_{-\infty}^{+\infty} d z n_{A}\left(\sqrt{z^{2}+\mathbf{b}^{2}}\right)$, is the optical thickness of a nucleus at an impact parameter $\mathbf{b}$, the nuclear matter density $n_{A}(\mathbf{r})$ is normalized as $\int d^{3} \mathbf{r} n(\mathbf{r})=A$. The leading-log contribution to $\left\langle\sigma^{2}\right\rangle_{\lambda}$ comes from the domain

$$
\frac{1}{Q^{2}} \lesssim r^{2} \lesssim r_{\lambda}^{2}
$$

where $r_{L}^{2}=1 / m_{s}^{2}$ and $r_{R}^{2}=1 / m_{c}^{2}$. Then,

$$
\begin{array}{r}
\left\langle\sigma^{2}\right\rangle_{L}=\left\langle\Psi_{L}\left|\sigma(x, r)^{2}\right| \Psi_{L}\right\rangle=\int d z d^{2} \mathbf{r}\left|\Psi_{L}(z, \mathbf{r})\right|^{2} \sigma^{2}(x, r) \\
\propto \frac{1}{Q^{2}} \int_{1 / Q^{2}}^{1 / m_{s}^{2}} \frac{d r^{2}}{r^{4}} \sigma^{2}(x, r) \propto \frac{1}{Q^{2} m_{s}^{2}}
\end{array}
$$


and, similarly,

$$
\left\langle\sigma^{2}\right\rangle_{R} \propto \frac{1}{Q^{2}} \int_{1 / Q^{2}}^{1 / m_{c}^{2}} \frac{d r^{2}}{r^{4}} \sigma^{2}(x, r) \propto \frac{1}{Q^{2} m_{c}^{2}} .
$$

Thus, there is a sort of filtering phenomenon, the target nucleus absorbs the $c \bar{s}$ Fock component of $W^{+}$with the helicity $\lambda=-1$, but is nearly transparent for $c \bar{s}$ states with opposite helicity $[5,6]$.

\section{Color dipole description of CC DIS off nuclei: first iteration of the $\mathbf{L L}(1 / x)$ evolution [7]}

We base our calculations of nuclear cross sections on the CD approach to the Leading $\log (1 / x)$ (LL $(1 / x)$ ), or BFKL [8], evolution of DIS [9, 10]. For nuclear targets a complete resummation of $\operatorname{LL}(1 / x)$ effects is as yet lacking. Our point is that at energies of the planned eIC [11], the $x$ dependence of nuclear shadowing (NS) is practically exhausted by the first $\operatorname{CD~} \operatorname{LL}(1 / x)$ iteration which is calculable exactly without invoking the large- $N_{c}$ approximation. Indeed, the first CD $\operatorname{LL}(1 / x)$ iteration dominates in the $x$ region

$$
\xi=\log \frac{x_{0}}{x} \lesssim \frac{1}{\Delta_{e f f}}
$$

where $\Delta_{\text {eff }} \simeq 0.1-0.2$ is the exponent of the local $x$-dependence of the proton structure function, $F_{2 p} \propto x^{-\Delta_{e f f}}[12]$.

When viewed in the laboratory frame, NS corrections to $F_{3}$ come from the coherent interaction of $c \bar{s}, c \bar{s} g, \ldots$ states. To the required accuracy, the Fock state expansion of the virtual $\mathrm{W}$ boson $\left|W^{*}\right\rangle$ reads

$$
\left|W^{*}\right\rangle=\sqrt{Z_{g}} \Psi_{\lambda}|c \bar{s}\rangle+\Phi_{\lambda}|c \bar{s} g\rangle
$$

where $\Psi_{\lambda}$ and $\Phi_{\lambda}$ are the light-cone wave functions (WF's) of the $c \bar{s}$ and $c \bar{s} g$ states $[4,5], \sqrt{Z_{g}}$ is the renormalization of the $c \bar{s}$ state by the virtual radiative corrections for the $c \bar{s} g$ state. For soft gluons the 3-parton WF takes the factorized form $\Phi_{\lambda}=\Psi_{\lambda}\left\{\Psi_{c g}-\Psi_{\bar{s} g}\right\}[9,10]$. The nuclear coherency condition reads

$$
\frac{x}{\beta} \lesssim x_{A}=\frac{1}{m_{N} R_{A}}=0.15 A^{-1 / 3}
$$

where $x=Q^{2} / 2 m_{N} v$ is the Bjorken variable, $v$ is the energy of the photon, $M$ is the invariant mass of the multiparton Fock state and $\beta=Q^{2} /\left(M^{2}+Q^{2}\right)$.

In the CD BFKL-Regge phenomenology of DIS one usually formulates the boundary condition at $x=x_{0}=0.03$ [13]. For extremely heavy nuclei $x_{A} \ll x_{0}$ and the $\operatorname{LL}(1 / x)$ evolution starts at $x=x_{A}$ with the boundary condition formulated in terms of the free-nucleon quantities $\operatorname{LL}(1 / x)$ evolved from $x_{0}$ down to $x_{A}$. At small $x$ satisfying the condition (2.3) the color dipoles $\left\{\mathbf{r}_{n}\right\}$ in the multiparton state are conserved in the interaction process. At the boundary $x=x_{A}$ and for $A \gg 1$, the nuclear S-matrices equal $[14,15]$

$$
\mathrm{S}_{n}\left(x_{A}, \mathbf{b},\{\mathbf{r}\}_{n}\right)=\exp \left[-\frac{1}{2} \sigma_{n}\left(x_{A},\{\mathbf{r}\}_{n}\right) T(\mathbf{b})\right],
$$




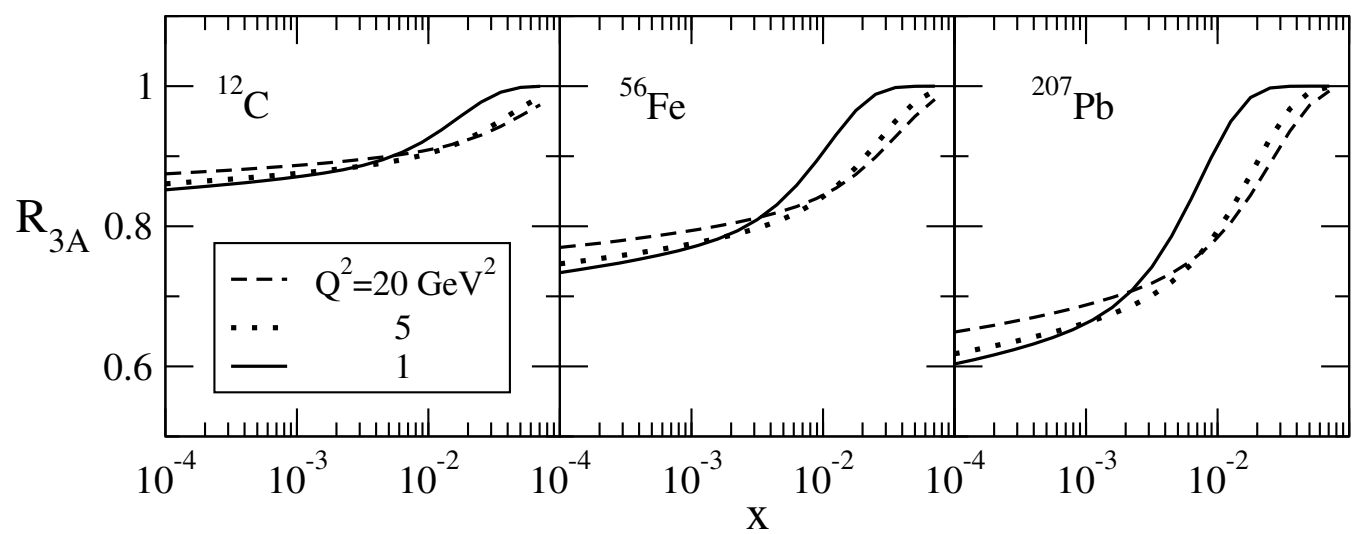

Figure 1: Predictions from $\mathrm{CD} L L(1 / x)$ evolution for $\mathrm{NS}$ ratio $R_{3 A}$ for $\mathrm{C}, \mathrm{Fe}$ and $\mathrm{Pb}$ nuclei as a function of $x$ at $Q^{2}=1,5,20 \mathrm{GeV}^{2}$.

where $\sigma_{n}\left(x_{A},\{\mathbf{r}\}_{n}\right)$ is the free-nucleon CD cross section for the $n$-parton state, and [16]

$$
\sigma_{2, A}\left(x_{A}, \mathbf{r}\right)=2 \int d^{2} \mathbf{b}\left[1-\mathrm{S}_{2}\left(x_{A}, \mathbf{b}, \mathbf{r}\right)\right] .
$$

Now one must evolve the nuclear S-matrix down to $x \ll x_{A}$. Specifically, after the gluon variables have been properly integrated out, the effect of the extra gluon in the Fock state expansion for the incident photon reduces to the renormalization of the S-matrix and nuclear cross section for the $c \bar{s} \mathrm{CD}[9,10]$,:

$$
\begin{aligned}
\frac{\partial \mathrm{S}_{2}(x, \mathbf{r} ; \mathbf{b})}{\partial \xi} & =\int d^{2} \rho|\psi(\rho)-\psi(\rho+\mathbf{r})|^{2}\left[\mathrm{~S}_{3}(x, \rho, \mathbf{r} ; \mathbf{b})-\mathrm{S}_{2}(x, \mathbf{r} ; \mathbf{b})\right] \\
\sigma_{A}(x, \mathbf{r}) & =\sigma_{2, A}\left(x_{A}, \mathbf{r}\right)+\sigma_{A}^{(1)}(x, \mathbf{r}) \\
\sigma_{A}^{(1)}(x, \mathbf{r}) & =2 \log \left(\frac{x_{A}}{x}\right) \int d^{2} \mathbf{b} \int d^{2} \rho|\psi(\rho)-\psi(\rho+\mathbf{r})|^{2} \\
& \times\left[\mathrm{S}_{2}\left(x_{A}, \mathbf{r} ; \mathbf{b}\right)-\mathrm{S}_{3}\left(x_{A}, \rho, \mathbf{r} ; \mathbf{b}\right)\right], \\
\sigma_{3}(x, \mathbf{r}, \rho) & =\frac{N_{c}^{2}}{N_{c}^{2}-1}\left[\sigma_{2}(x, \rho)+\sigma_{2}(x, \rho+\mathbf{r})\right]-\frac{1}{N_{c}^{2}-1} \sigma_{2}(x, \mathbf{r})
\end{aligned}
$$

where $\rho$ is the size of the $c g$ dipole and

$$
\psi(\rho)=\frac{\sqrt{C_{F} \alpha_{S}}}{\pi} \cdot \frac{\rho}{\rho^{2}} \cdot \frac{\rho}{R_{c}} K_{1}\left(\frac{\rho}{R_{C}}\right)
$$

is the radial WF of the $c g$ state with the Debye screening of infrared gluons $[9,10]$. The absorption cross section is an expectation value

$$
\sigma_{A, \lambda}\left(x, Q^{2}\right)=\left\langle\Psi_{\lambda}\left|\sigma_{A}(x, \mathbf{r})\right| \Psi_{\lambda}\right\rangle .
$$

The shadowing ratio $R_{3 A}$, equals

$$
R_{3 A}=\frac{\sigma_{A, L}-\sigma_{A, R}}{A\left(\sigma_{L}-\sigma_{R}\right)}=1-\frac{\delta \sigma_{A, L}-\delta \sigma_{A, R}}{A \sigma_{L}-A \sigma_{R}},
$$


where $\sigma_{\lambda}\left(x, Q^{2}\right)=\sigma_{2, \lambda}\left(x_{A}, Q^{2}\right)+\log \left(x_{A} / x\right) \sigma_{\lambda}^{(1)}\left(x_{A}, Q^{2}\right)$ is the $\operatorname{LL}(1 / x)$-evolved free-nucleon cross section. Hence, the nuclear shadowing correction $\left(1-R_{3 A}\right) \cdot x F_{3}^{v(\bar{v})}$ which should be added to $x F_{3}^{v(\bar{v})}$ extracted from the $v(\bar{v}) F e$ data to get the "genuine" $x F_{3}^{v(\bar{v})}$. This correction positive-valued and does increase $x F_{3}^{v(\bar{v})}$ of the impulse approximation.

Our interest is in the well evolved shadowing at $x \ll x_{A}$, the onset of NS at $x \gtrsim x_{A}$ must be treated within the light-cone Green function technique [17, 18], here we show only the gross features of the large- $x$ suppression of NS following the prescriptions from Refs. [16, 19] and the $\beta$-dependence of the large rapidity gap DIS as predicted in [20] and confirmed in the HERA experiments [21]. We use the free-nucleon $C D$ cross section tested against the experimental data from HERA, it is described in [7]. We calculate $R_{3 A}$ as a function of $x$ for several values of $Q^{2}$. Our results obtained for realistic nuclear densities of Ref.[22] are presented in Figure 1. Shown is the ratio $R_{3 A}$ for different nuclear targets including ${ }^{56} \mathrm{Fe}$. At small $x$ and high $Q^{2}$ the shadowing correction scales, $\delta \sigma_{L, R} \propto 1 / Q^{2}$. The absorption cross section $\sigma_{L, R}$ scales as well. The ratio $\delta \sigma_{L, R} / \sigma_{L, R}$ slowly decreases with growing $Q^{2}$ because of the logarithmic scaling violation in $\sigma_{L, R}$. Toward the region of $x>0.01$, both the nuclear form factor and the mass threshold effect suppress the shadowing correction $1-R_{3 A}$ at $Q^{2} \lesssim\left(m_{c}+m_{s}\right)^{2}$ (see Fig.1).

\section{Summary}

The parity non-conservation effect in diffractive charged current DIS was quantified in terms of color dipole sizes of left-handed and right-handed electroweak bosons. We identified the origin and estimated the strength of the left-right asymmetry effect. Based on the color dipole approach which correctly reproduces the experimental data on the proton structure function measured at HERA and the NMC data on nuclear shadowing, we reported the quantitative predictions for the $\operatorname{LL}(1 / x)$ evolution of the nuclear shadowing effect in absorption of left-handed and right-handed $W$-bosons.

\section{Acknowledgments:}

The author is thankful to Roberto Fiore for the invitation to attend the Workshop and to all organizers for hospitality. Special thanks to Alessandro Papa for careful reading the manuscript and useful remarks. The work has been partly supported by the DFG grant 436 RUS 17/82/06 and by the RFBR grant 06-02-16905-a.

\section{References}

[1] B.L. Ioffe, V.A.Khoze and L.N. Lipatov, Hard Processes, v.1, North Holland 1984.

[2] N.N. Nikolaev and B.G. Zakharov, Z. Phys. C49 (1991) 607; C53 (1992) 331; C64 (1994) 631.

[3] A.H. Mueller, Nucl. Phys. B415 (1994) 373; A.H. Mueller and B. Patel, Nucl. Phys. B425 (1994) 471.

[4] R. Fiore and V.R. Zoller, JETP Lett. 82 (2005) 385.

[5] R. Fiore and V.R. Zoller, Phys. Lett. B632 (2006) 87.

[6] V.R. Zoller, J. Phys. G32 (2006) 2305. 
[7] N.N. Nikolaev, W. Schafer, B.G. Zakharov and V.R. Zoller, Unitarity constraints for DIS off nuclei: predictions for electron-ion colliders, hep-ph/0610319.

[8] E.A. Kuraev, L.N. Lipatov and V.S. Fadin, Sov. Phys. JETP 45 (1977) 199 [Zh. Eksp. Teor. Fiz. 72 (1977) 377]; I.I. Balitsky and L.N. Lipatov, Sov. J. Nucl. Phys. 28 (1978) 822 [Yad. Fiz. 28 (1978) 1597].

[9] N.N. Nikolaev, B.G. Zakharov and V.R. Zoller, JETP Lett. 59 (1994) 6 [Pisma Zh. Eksp. Teor. Fiz. 59 (1994) 8]; Phys. Lett. B328, 486 (1994).

[10] N.N. Nikolaev and B.G. Zakharov, J. Exp. Theor. Phys. 78 (1994) 598 [Zh. Eksp. Teor. Fiz. 105 (1994) 1117]; Z. Phys. C64 (1994) 631.

[11] A. Deshpande, R. Milner, R. Venugopalan and W. Vogelsang, Ann. Rev. Nucl. Part. Sci. 55 (2005) 165 and references therein.

[12] J. Breitweg et al. [ZEUS Collaboration], Eur. Phys. J. C7 (1999) 609; C. Adloff et al. [H1 Collaboration], Phys. Lett. B520 (2001) 183.

[13] N. N. Nikolaev, B. G. Zakharov and V. R. Zoller, JETP Lett. 66138 (1997) 138 [Pisma Zh. Eksp. Teor. Fiz. 66 (1997) 134]; N. N. Nikolaev, J. Speth and V. R. Zoller, Phys. Lett. B473 (2000) 157; J. Exp. Theor. Phys. 93 (2001) 957 [Zh. Eksp. Teor. Fiz. 93 (2001) 1104]; Eur. Phys. J. C22 637 (2002) 637.

[14] R. J. Glauber, in Lectures in Theoretical Physics, edited by W. E. Brittin et al. (Interscience Publishers, Inc., New York, 1959), Vol. 1, p. 315; R. J. Glauber and G. Matthiae, Nucl. Phys. B21 (1970) 135.

[15] V. N. Gribov, Sov. Phys. JETP 29 (1969) 483 [Zh. Eksp. Teor. Fiz. 56 (1969) 892].

[16] N.N. Nikolaev and B.G. Zakharov, Z. Phys. C49 (1991) 607; V. Barone, M. Genovese, N. N. Nikolaev, E. Predazzi and B. G. Zakharov, Z. Phys. C58 541 (1993) 541.

[17] B.G. Zakharov, Phys. Atom. Nucl. 61 (1998) 838 [Yad. Fiz. 61 (1998) 924].

[18] B.G. Zakharov, JETP Lett. 63 (1996) 952 [Pisma Zh. Eksp. Teor. Fiz. 63 (1996) 906]; Ibid, 64 (1996) 781 [Pisma Zh. Eksp. Teor. Fiz. 64 (1996) 737].

[19] V. A. Karmanov and L. A. Kondratyuk, JETP Lett. 18 (1973) 266 [Pisma Zh. Eksp. Teor. Fiz. 18 (1973) 451].

[20] N. Nikolaev and B. G. Zakharov, Z. Phys. C53 (1992) 331. M. Genovese, N. N. Nikolaev and B. G. Zakharov, J. Exp. Theor. Phys. 81 (1995) 625 [Zh. Eksp. Teor. Fiz. 108 (1995) 1141]; Phys. Lett. B380 (1996) 213.

[21] S. Chekanov et al. [ZEUS Collaboration], Nucl. Phys. B713 (2005) 3 and references therein.

[22] H. de Vries, C.W. De Jager and C. de Vries, Atomic Data and Nuclear Data Tables 36 (1987) 495. 